\title{
Motivação à prática de atividades físicas: um estudo com praticantes não-atletas ${ }^{1}$
}

\author{
Marina Pereira Gonçalves ${ }^{2}$ - Universidade Federal do Rio Grande do Norte, Natal, Brasil \\ João Carlos Alchieri - Universidade Federal do Rio Grande do Norte, Natal, Brasil
}

\begin{abstract}
Resumo
Este estudo objetivou verificar a motivação para praticar atividades físicas (AF) entre praticantes não-atletas da cidade de Natal-RN. Para tanto, contou-se com a colaboração de 309 praticantes de AF, com idades entre 16 e 74 anos, distribuídos equitativamente quanto ao sexo. Estes responderam à Escala de Motivação à Prática de Atividades Físicas Revisada (MPAM-R), que possui cinco fatores: Diversão, Saúde, Aparência, Competência e Social, e questões sociodemográficas. No geral, os participantes deste estudo demonstraram praticar AF mais por questões de Saúde, verificando-se uma maior média no fator Saúde para mulheres e idosos; maior média no fator Aparência para os praticantes de exercícios e maior média no fator Social entre aqueles que praticam AF acompanbados. Conclui-se que os objetivos da presente pesquisa foram alcançados, tais achados possibilitam uma maior compreensão acerca do que motiva as pessoas a praticar atividade física. Entretanto, sugere-se a realização de novos estudos considerando outras variáveis.

Palavras-chave: Motivação, Atividade física, Praticantes não-atletas.
\end{abstract}

\section{Motivation to practicing physical activities: a study with non-athletes}

\begin{abstract}
The study aimed at investigating the motivation to practicing physical activities (PA) among non-atlhetes in the city of Natal/RN. 309 practitioners of PA participated in the survey, aging between 16 and 74 years of age, equally distributed by gender. They answered the Revised Motivation for Physical Activity Measure (MPAM-R) that has five factors: Enjoyment, Health, Appearance, Competence and Social, and socio-demographic questions. In general, the main motive presented by the participants to practice PA was Health. It was verified yet, that women and the elderly had a higher average for the Health factor; among the exercise practitioners, a higher average in the Appearance factor was found; and a higher average in the Social factor was found among those that practice $P A$ with accompaniment. It is concluded that the objectives of this study were achieved; these findings enable a better understanding of what motivates people to perform physical activity. However, further studies are suggested considering other variables.

Keywords: Motivation, Physical activity, Practitiones no atletics.
\end{abstract}

O estudo da motivação no contexto da atividade física e do esporte tem sido alvo de parte da literatura produzida na área da Psicologia do Esporte. Entretanto, alguns estudos têm buscado verificar a motivação em atletas de alto rendimento que precisam estar motivados para alcançar seus objetivos, conquistar recordes e vitórias. Outras pesquisas centram-se na compreensão dos fatores e processos associados à adesão, persistência e abandono da atividade física regular, buscando identificar o que motiva as pessoas "não-atletas" a praticar atividade física regularmente e, assim, deixar de ser indivíduos sedentários (Biddle, 1997; Dishman, 1994; Gauvin

\footnotetext{
1 Artigo elaborado a partir da dissertação de M. P. Gonçalves, intitulada "Adaptação e validação da escala de motivação à prática de atividades físicas". Universidade Federal do Rio Grande do Norte, Natal, 2008. Os autores agradecem o apoio financeiro da Capes para a realização desta pesquisa.

2 Endereço para correspondência:

Avenida Pombal, 1542, apto. 202 - Manaíra - 58038-242 - João Pessoa-PB

E-mail: marinapgoncalves@gmail.com
}

\& Spence, 1995; Gouveia, 2001). Nesse sentido, a presente pesquisa busca investigar a motivação à prática de atividades físicas em uma amostra de praticantes "não-atletas".

A atividade física (AF) é definida como "todo movimento corporal produzido pela musculatura esquelética, que resulte em um gasto energético maior do que os níveis de repouso" (Caspersen, Powell \& Christenson, 1985, p. 16). Nessa definição percebe-se que a AF engloba os exercícios físicos e os esportes, mas também o equivalente em gastos de energia em outros tipos de atividades, como lazer ativo, trabalho ocupacional e tarefas domésticas (Miragaya, 2006). O presente estudo irá investigar a motivação para praticar AF, considerando como AF regular a prática de algum tipo de exercício físico ou esporte.

Os benefícios alcançados pela prática regular de AF são inúmeros. Segundo a Organização Mundial de Saúde (2006), esta prática reduz o risco de mortes prematuras, doenças do coração, acidente vascular cerebral, câncer de cólon e mama e diabetes tipo II. Atua na 
prevenção ou redução da hipertensão arterial, previne o ganho de peso (diminuindo o risco de obesidade), auxilia na prevenção ou redução da osteoporose, promove bem-estar, reduz o estresse, a ansiedade e a depressão.

Como afirma Miragaya (2006), há evidências científicas apontando que a prática de AF é uma ferramenta essencial para a promoção da saúde, porque ela inibe o surgimento e o desenvolvimento de fatores de risco que predispõem ao aparecimento de disfunções crônico-degenerativas. Vários estudos epidemiológicos têm demonstrado que indivíduos fisicamente ativos vivem mais que indivíduos sedentários e têm menor propensão a desenvolver vários tipos de doenças crônicas.

Entretanto, apesar de ser considerável o número de estudos (Andreotti \& Okuma, 2003; Epiphanio, 1999; Ferrareze, 1997; Mello, Boscolo, Esteves \& Tufik, 2005; Miragaya, 2006) que apontam a importância da prática de atividades físicas na manutenção da saúde, no aumento do bem-estar e da qualidade de vida em geral, tais razões não parecem ser suficientes para levar indivíduos sedentários a praticarem e a se manterem praticando alguma AF por um período superior a seis meses (Robertson \& Mutrie, 1989, citados por Andreotti \& Okuma, 2003).

De acordo com o Conselho Federal de Educação Física (2004), a evolução da indústria de bens de consumo tem deixado a vida cada vez mais confortável, de modo que o aparato tecnológico e informacional contribui para que, a cada dia, as pessoas façam menos movimento, aumentando, consequentemente, o risco ao sedentarismo. O sedentarismo, caracterizado pela ausência de AF regular, atualmente é considerado tão prejudicial à saúde quanto qualquer outro tipo de doença, podendo acarretar um custo econômico a médio e longo prazo, para o indivíduo, a família e para a sociedade (CONFEF, 2004). Nesse sentido, pesquisas sobre motivação à prática de AF tornam-se relevantes, a fim de viabilizar futuros estudos que visam minimizar o sedentarismo entre a população.

A motivação, um processo psicológico básico que auxilia na compreensão das diferentes ações e escolhas individuais, é um dos fatores determinantes do modo como uma pessoa se comporta (Schultz \& Schultz, 2002). E apesar da quantidade considerável de teorias e perspectivas na busca por compreender a motivação humana (ver Allport, 1961; Bandura, 1977; Kelly, 1955; Maslow, 1970; McClelland, 1955; Murray, 1967;
Rotter, 1990, citados por Schultz \& Schultz, 2002), no presente estudo, dar-se-á ênfase a Teoria da Autodeterminação (Deci \& Ryan, 1985), por possuir representatividade no contexto da atividade física, tendo sido utilizada em diversas pesquisas da área (Fernandes, 2003; Fernandes \& Vasconcelos-Raposo, 2005; Moreno, Cervelló \& González-Cutre, 2006; Ntoumanis, 2001; Frederick \& Ryan, 1993; Ryan, Frederick, Lepes, Rubio \& Sheldon, 1997, entre outros).

A Teoria da Autodeterminação - TAD é uma macroteoria da motivação humana que tem relação com o desenvolvimento e o funcionamento da personalidade dentro dos contextos sociais. Essa teoria analisa o grau em que as condutas humanas são volitivas ou autodeterminadas, isto é, o quanto as pessoas realizam suas ações em um nível maior de reflexão e se comprometem com essas ações de forma voluntária, por sua própria escolha. Dessa forma, o indivíduo pode ser motivado intrínseca ou extrinsecamente para tentar satisfazer suas necessidades e, assim, atingir a autodeterminação (Deci \& Ryan, 1985; Ryan \& Deci, 2000).

No contexto da atividade física, a TAD serviu de respaldo teórico para o desenvolvimento de alguns instrumentos de medida com a finalidade de investigar e medir a motivação à prática de atividades físicas. Um desses instrumentos é a escala Motives for Physical Activity Measure - MPAM, de Frederick e Ryan (1993), que mede três motivos para se praticar AF: Interesse/Diversão; Competência e Motivos relacionados com o corpo. Posteriormente, a revisão desta escala (Motives for Physical Activity Measure-Revised - MPAM-R) foi feita por Ryan e cols. (1997) e a mesma passou a medir cinco motivos: 1) Diversão, onde quer ser fisicamente ativo porque considera a atividade divertida, o torna feliz, é interessante, estimulante e agradável; 2) Competência, que visa praticar atividade física para ser melhor naquela atividade, encontrar desafios e adquirir novas habilidades; 3) Aparência, em que busca a atividade para se tornar mais atraente, ter músculos definidos e alcançar ou manter um peso desejado; 4) Saúde, que refere-se a ser fisicamente ativo para ter saúde, força e energia; e 5) Social, onde se busca praticar atividade física para estar com os amigos e conhecer novas pessoas.

Os achados de algumas pesquisas que utilizaram as escalas MPAM e MPAM-R são apresentados a seguir. Frederick e Ryan (1993), contando com a participação de 376 adultos 
americanos praticantes de esportes individuais (tênis, iatismo) e exercícios físicos (correr, aeróbica) e utilizando a MPAM, identificaram que os praticantes de esporte pontuaram mais alto nos motivos de diversão e competência, enquanto os praticantes de exercícios apresentaram médias maiores nos motivos relacionados ao corpo.

Já na pesquisa realizada por Ryan e cols. (1997), com uma amostra composta por 40 estudantes de uma universidade americana, advindos de programas voluntários de AF (não ofereciam créditos aos participantes), sendo 24 praticantes de tae kwon do e 16 praticantes de aeróbica, estes responderam a escala MPAM e algumas variáveis sobre abandono e frequência da atividade. Os resultados indicaram que os praticantes de tae kwon do (esporte) apresentaram motivos relacionados com competência e diversão, enquanto os praticantes de aeróbica (exercício físico) apresentaram motivos relacionados com a aparência, não havendo diferença significativa no nível de adesão dos dois grupos.

Em um segundo estudo, desta vez utilizando a versão revisada da Escala de Motivação à Prática de Atividades Físicas (MPAM$\mathrm{R})$, esses mesmos autores identificaram que a adesão foi associada com motivos centrados em diversão, competência e interação social, mas não com motivos de aparência. E no que se refere às diferenças de gênero, verificou-se que as mulheres pontuaram mais alto que os homens nos fatores aparência e saúde, e ambos, homens e mulheres, relataram a mesma ordem de significância para os cinco fatores: saúde, aparência, competência, diversão e social.

Em uma pesquisa realizada na Espanha por Moreno, Cervelló e Martínez (2007), estes autores apresentaram a validação da MPAM-R para o espanhol e buscaram comprovar os efeitos dos cinco motivos sobre o gênero, a idade, a forma da atividade, o tempo e os dias de prática contando com a participação de 561 adultos praticantes de atividades físicas não competitivas. Os resultados indicaram que as pessoas de maior idade deram mais importância aos motivos relacionados com a saúde, enquanto os mais jovens priorizaram os motivos relacionados com a aparência. Em relação ao gênero, as mulheres deram menos importância aos motivos de competência do que os homens. As pessoas que praticavam por mais tempo apresentaram motivos de diversão e saúde, e os que praticavam há mais dias apontaram motivos de saúde. Os sujeitos que praticavam em programas orientados mostraram motivos relacionados ao social, à saúde e à competência.

Outras pesquisas sobre motivação à prática de AF também foram conduzidas, no entanto sem utilizar as escalas MPAM ou MPAM-R como instrumento de investigação. Por exemplo, na pesquisa de Hellín, Moreno e Rodriguez (2004), esses autores contaram com a participação de 1107 praticantes de AF da região de Murcia na Espanha, com idades entre 15 e 64 anos, e identificaram que os sujeitos mais jovens tinham uma maior inclinação para as competições e uma maior preocupação com a imagem corporal e a estética, enquanto os sujeitos com idade mais avançada praticavam por motivos lúdicos, relaxantes e de relação. Em relação ao gênero, esses mesmos autores encontraram que as mulheres apresentaram uma maior preocupação com a imagem corporal e a estética do que os homens.

Já em uma pesquisa realizada com estudantes universitários americanos por Kilpatrick, Hebert e Bartholomew (2005), esses autores identificaram que os homens apresentaram uma maior motivação acerca do desafio, competição, força, resistência e reconhecimento social, enquanto as mulheres apresentaram maior motivação na variável de controle do peso, e ambos, homens e mulheres, reconheceram a importância da AF para um estado de saúde positivo. Esses mesmos autores também verificaram que existia uma maior motivação entre praticantes de exercício, para aspectos como força, resistência, aparência, controle do estresse e controle do peso, enquanto os praticantes de esporte apresentaram maior motivação para aspectos como afiliação, desafio, competição e reconhecimento social.

No Brasil, não foram encontradas pesquisas que tenham utilizado as escalas MPAM ou MPAM-R para identificar motivos de adesão à prática de AF, entretanto alguns estudos com praticantes não-atletas foram conduzidos, por exemplo, por Andreotti e Okuma (2003), com a participação de 44 idosos que iniciavam a prática de $A F$, que verificaram que as principais razões para a adesão inicial desses indivíduos em um programa de AF foram: indicação de amigos, crença nos benefícios da AF para saúde, indicação médica e busca de convívio social. Já Santos e Knijnik (2006), num estudo com 30 adultos, com idades entre 40 e 60 anos, identificaram que os principais motivos iniciais de adesão à prática de AF foram: ordem médica; lazer e qualidade de vida; estética e saúde (ou condicionamento físico). 
Diante do exposto, e buscando acrescentar dados à literatura brasileira sobre motivação à prática de atividades físicas, a presente pesquisa objetivou verificar as médias dos participantes nos cincos motivos (diversão, competência, aparência, saúde e social) da escala MPAM-R, em relação às variáveis sociodemográficas: idade, gênero, IMC (índice de massa corporal), tipo de AF (exercício ou esporte), forma de praticar (sozinho ou acompanhado) e o tempo de prática. O método utilizado para atingir tal objetivo é apresentado a seguir.

\section{Método}

Amostra

A amostra foi de tipo acidental (de conveniência) e não-probabilística, sendo incluídos os participantes que concordaram em fazer parte do estudo e que não eram atletas profissionais. Nesse sentido, contou-se com a colaboração de 309 praticantes de atividades físicas, classificadas em exercícios físicos (musculação, ginástica, caminhada, hidroginástica e dança) e esportes (futebol, vôlei, basquete, natação, artes marciais, tênis e ciclismo), da cidade do Natal-RN. Estes tinham idades variando entre 16 e 74 anos $(M=35$ anos; $D P=14,11)$, distribuídos equitativamente quanto ao sexo, sendo 152 homens $(49,2 \%)$ e 157 mulheres $(50,8 \%)$.

\section{Instrumentos}

Escala de Motivação à Prática de Atividades Físicas Revisada (MPAM-R)

Esta escala mede cinco motivos para se praticar AF: diversão, competência, aparência, saúde e social, cujos parâmetros psicométricos são apresentados por Ryan e cols. (1997), indicando índices de consistência interna (alfas) de 0,92; 0,$91 ; 0,88 ; 0,78$ e 0,83 , respectivamente. A adaptação e validação dessa escala para o espanhol podem ser vistas em dois estudos recentes, um na Colômbia, por Celis-Merchán (2006), e outro na Espanha, por Moreno e cols. (2007). Entretanto, nenhuma pesquisa foi encontrada no Brasil em que esta escala tenha sido empregada, demandando que se comprove sua adequação. Para tanto, sua adaptação e validação foi realizada para o contexto brasileiro por Gonçalves (2008). A versão em português da MPAM-R ficou composta por 26 itens, sendo exemplificados a seguir: 1) Diversão: "Porque acho essa atividade estimulante"; "Porque essa atividade me faz feliz"; 2) Competência: "Porque gosto do desafio"; "Para adquirir novas habilidades físicas"; 3) Aparência: "Para definir meus músculos e ter uma boa aparência"; "Porque quero ser atraente para os outros"; 4) Saúde: "Porque quero ser fisicamente saudável"; "Para ter mais energia"; e 5) Social: "Para estar com meus amigos"; "Porque quero conbecer novas pessoas". Os índices de consistência interna (alfas) para cada um dos fatores foram de 0,$88 ; 0,85 ; 0,79 ; 0,84$ e 0,75 , respectivamente. Os participantes responderam à questão "Pratico atividade física..." por meio dos itens que compõem a escala, sendo estes exemplificados acima. Esses itens são respondidos por uma escala tipo Likert de sete pontos (1=Discordo totalmente a $7=$ Concordo Totalmente).

\section{Questões sociodemográficas}

Foram elaboradas questões de caráter sociodemográfico (por exemplo, idade, sexo, estado civil, IMC e escolaridade) e outras que permitissem avaliar a atividade física realizada por esses participantes (atividade que pratica, tempo de prática, horas de prática por semana, entre outras).

\section{Procedimento}

Todos os dados foram coletados na cidade do Natal-RN, em alguns locais onde as pessoas praticam AF (por exemplo: calçadão, parques, academias e clubes). Para a realização da coleta de dados em locais privados, tais como clubes ou academias, inicialmente entrou-se em contato com os gerentes ou administradores desses estabelecimentos solicitando permissão para abordar seus clientes a responderem o questionário. Já em locais públicos, as pessoas foram diretamente abordadas por pesquisadores previamente treinados, sendo solicitadas a responderem o questionário. Destaca-se que, para cada participante, foram explicados previamente os objetivos da pesquisa, bem como da sua participação voluntária, assegurando-lhes o anonimato e a confidencialidade de suas respostas. Em média, 10 minutos foram suficientes para concluir sua participação.

\section{Análises dos dados}

A tabulação dos dados, as estatísticas descritivas (medidas de tendência central e dispersão) e as análises de variância (ANOVA e MANOVA) foram realizadas por meio do SPSSWIN 15 (Statistical Package for the Social Science). 


\section{Resultados e discussão}

Com a finalidade de testar o objetivo proposto nesta pesquisa, procurou-se cumprir dois procedimentos específicos: 1- caracterizar os participantes verificando suas pontuações médias em relação à escala de resposta do instrumento utilizado (MPAM-R) e 2- verificar se os participantes se diferenciavam no construto estudado em função do sexo, idade, índice de massa corporal (IMC), tipo de atividade física (exercício ou esporte), forma de praticar (sozinho ou acompanhado) e o tempo de prática.

A escala de motivação utilizada varia de $1=$ Discordo totalmente a $7=$ Concordo totalmente, avaliando assim o grau de discordância/concordância dos participantes nos motivos para se praticar atividade física. No que se refere ao primeiro procedimento antes descrito, realizou-se uma MANOVA para medidas repetidas a fim de verificar se as médias dos participantes nos cinco fatores de motivação apresentavam diferenças estatisticamente significativas em relação a mediana teórica da escala (4).

Os resultados demonstraram que foi observado um efeito multivariado entre os fatores de motivação e o ponto médio teórico da escala de resposta [Lamda de Wilks=0,174; $\mathrm{F}(4,000)=355,109, \mathrm{p}<0,001]$. O teste de Post Hoc realizado demonstrou índices de significância adequados (intervalo de confiança ajustado: Bonferroni; $p<0,05)$, para as diferenças de média obtidas. Dessa forma, os participantes apresentaram motivos de Diversão $(M=5,49$, $D P=1,05 ; \quad p<0,001) ;$ Saúde $(M=6,32, \quad D P=0,70$; $p<0,01)$; Aparência $(M=4,72, D P=1,29 ; p<0,05)$ e Competência $(M=4,36, D P=1,62 ; p<0,05)$ maiores do que o ponto médio da escala (4). Entretanto, no fator Social $(M=3,60, \quad D P=1,29 ; \quad p<0,05)$ verificou-se que a média dos participantes foi estatisticamente inferior ao ponto mediano da escala. Em relação a esse resultado, verificou-se que para os participantes desse estudo em geral, o motivo principal para praticar AF foi a preocupação com a saúde, o que corrobora estudos anteriores (Hellín \& cols., 2004; Howley \& Don Franks, 2000; Kilpatrick \& cols., 2005; Moreno \& cols., 2007; Ryan \& cols., 1997). Ademais, de acordo com Fernandes (2003), nesses casos o comportamento do indivíduo é motivado pela apreciação dos resultados e benefícios da participação numa atividade. Por exemplo, quando se busca a atividade física com ênfase na prevenção de doenças ou melhoria da condição física, o indivíduo pode considerar a atividade desagradável ou desinteressante, mas ainda assim se motiva pelo benefício que ela causa, sendo esta considerada uma motivação extrínseca.

Dando continuidade, em seguida, realizouse uma MANOVA, objetivando verificar se os participantes apresentariam diferenças estatisticamente significativas nos fatores de motivação (diversão, saúde, aparência, competência e social), considerando como variáveis independentes o gênero (masculino e feminino); a faixa etária (jovens, adultos jovens, adultos intermediários e idosos) e o índice de massa corporal - IMC (baixo peso, normal, obesidade graus I, II e III), sendo estas variáveis características dos participantes da amostra. Essa técnica estatística permite verificar a relação entre diversas categorias de variáveis independentes discretas (VI's) em relação a diversas categorias de variáveis dependentes métricas (VD's) (Hair, Anderson, Tatham \& Black, 2003; Pérez, 2001; Tabachnick \& Fidel, 2001). A seguir, foram dispostos na Tabela 1 os resultados da MANOVA indicando as diferenças de média estatisticamente significativas.

Tabela 1 - Análise de variância da motivação em função do sexo, idade e IMC

\begin{tabular}{ccccccc}
\hline Variáveis & MANOVA & \multicolumn{5}{c}{ ANOVA } \\
\cline { 3 - 6 } & & DI & SD & AP & CP & SC \\
\hline Sexo $(S)$ & $F(5,265)$ & $F(1,269)$ & $F(1,269)$ & $F(1,269)$ & $F(1,269)$ & $F(1,269)$ \\
& 2,05 & 0,07 & $4,93^{*}$ & 0,00 & 1,87 & 1,65 \\
Idade $(I)$ & $F(15,732)$ & $F(3,269)$ & $F(3,269)$ & $F(3,269)$ & $F(3,269)$ & $F(3,269)$ \\
& 1,35 & 1,18 & $5,10 * *$ & 0,47 & 0,08 & 0,08 \\
$I M C$ & $F(20,880)$ & $F(4,269)$ & $F(4,269)$ & $F(4,269)$ & $F(4,269)$ & $F(4,269)$ \\
& 1,28 & 1,37 & 1,60 & 1,36 & 1,36 & 0,68 \\
$S \times I \times I M C$ & 1,23 & 0,91 & 2,02 & 0,83 & 0,65 & 0,63 \\
\hline
\end{tabular}

Notas: MANOVA $=$ análise de variância multivariada; ANOVA $=$ análise de variância univariada; $* * * p<0,001$; Notas: DV:

Diversão; SD: Saúde; AP: Aparência; CP: Competência e SC: Social

Como pode ser observada na tabela acima, a diferença de média verificada para as variáveis sexo e idade em relação ao fator saúde foi a única estatisticamente significativa. Assim, de fato, na 
motivação para praticar atividade física verificouse que as mulheres $(M=6,43, D P=0,11)$ praticavam mais por questões de saúde do que os homens $(M=6,12, D P=0,09)$. A preocupação com a saúde atribuída mais às mulheres pode ser explicada por Boltanski, 1979; Lins, 1999; Messner, 1995, citados por Salles-Costa, Heilborn, Werneck, Faerstein e Lopes (2003), os quais consideram que a inserção da prática de atividades físicas no universo feminino está associada à manutenção da saúde e valorização dos cuidados com a imagem corporal, ressaltando-se que as mulheres apresentam uma percepção mais sensível do corpo e por isso buscam mais praticar atividades físicas do que o homens. Atualmente a prática de atividades físicas no universo feminino ganha grande proporção por meio da busca do corpo delineado e controle do peso corporal, ressaltando-se que em comparação a qualquer outro período, as mulheres estão gastando muito mais tempo com o tratamento e a disciplina dos seus corpos (Salles-Costa \& colaboradores, 2003).

No que se refere à variável idade, os idosos $(M=6,52, D P=0,14)$ apontaram praticar atividade física mais por motivos de saúde do que os jovens $(M=6,34, \quad D P=0,16), \quad$ adultos jovens $(M=5,85$, $D P=0,14)$ e adultos $(M=6,44, D P=0,10)$. Esse resultado seria de se esperar, tendo em vista que com o aumento da idade as pessoas tornam-se mais suscetíveis a certos tipos de doença e, com isso, a prática de AF regular passa a ser recomendada pelos médicos como forma de tratamento ou controle de algumas doenças. Nessa perspectiva, estudos como os de Andreotti e Okuma (2003) e Santos e Knijnik (2006) revelaram que a indicação médica foi relatada pelos participantes, adultos e idosos como um dos principais motivos para se iniciar a prática de AF. Esses resultados também são coerentes com a literatura, como por exemplo no estudo realizado por Moreno, Cervelló e Martínez (2007), em que as pessoas de maior idade deram mais importância aos motivos relacionados com a saúde, enquanto os mais jovens priorizaram os motivos relacionados com a aparência.

No que se refere ao índice de massa corporal, não foram encontrados resultados estatisticamente significativos, no entanto, ressaltase a importância de estudos futuros que verifiquem a relação da motivação com a prática de atividade e a obesidade, por exemplo, tendo em vista que em alguns estudos (por exemplo,
Kilpatrick \& cols., 2005) a variável de controle do peso tem sido identificada como relevante para se compreender a motivação à prática de $\mathrm{AF}$. Ademais, conhecendo-se as principais motivações das pessoas com sobrepeso ou obesas, seria de extrema relevância para viabilizar estratégias de intervenção que visem diminuir o sedentarismo entre essas pessoas. Por último, como visto na tabela acima, verificou-se que o efeito de interação entre sexo, idade e IMC não foi estatisticamente significativo, o que indica que no presente estudo, essas variáveis não interagem.

Dando continuidade, realizou-se outra MANOVA, objetivando agora verificar se os participantes apresentariam diferenças estatisticamente significativas nos fatores de motivação (diversão, saúde, aparência, competência e social), considerando como variáveis independentes: o tipo de AF (exercício ou esporte), a forma de praticar (sozinho ou acompanhado) e o tempo de prática (um a seis meses, sete meses a um ano, mais de um ano), sendo essas variáveis características da $\mathrm{AF}$ praticada pelos participantes da amostra. A seguir, foram dispostos na Tabela 2 os resultados da MANOVA indicando as diferenças de média que foram estatisticamente significativas.

Como pode ser observada na tabela abaixo, a diferença de média verificada para as variáveis tipo de AF e forma de praticar foi a única estatisticamente significativa. Assim, de fato, na motivação para praticar atividade física verificouse que os praticantes de exercício $(M=5,08$; $D P=0,15)$ praticavam mais por questões de aparência do que as praticantes de esporte $(M=4,57$; $D P=0,20)$. Tais resultados também corroboram com os resultados verificados na literatura (Frederick \& Ryan, 1993; Ryan \& cols., 1997), apontando que as pessoas que praticam exercícios o fazem mais por motivos de aparência do que os que praticam esportes, e dependendo do tipo da atividade praticada, as pessoas podem apresentar diferentes tipos de motivação. Ressalta-se ainda que Kilpatrick e cols. (2005) verificaram em seus estudos que existia uma maior motivação entre praticantes de exercício para aspectos como força, resistência, aparência, controle do estresse e controle do peso, enquanto para os praticantes de esporte havia uma maior motivação para aspectos como afiliação, desafio, competição e reconhecimento social. 
Tabela 2 - Análises de variância da motivação em função do tipo de AF, forma e tempo de prática

\begin{tabular}{|c|c|c|c|c|c|c|}
\hline \multirow[b]{2}{*}{ Variáveis } & \multirow[b]{2}{*}{ MANOVA } & \multicolumn{5}{|c|}{ ANOVA } \\
\hline & & $\mathrm{DI}$ & SD & $\mathrm{AP}$ & $\mathrm{CP}$ & SC \\
\hline \multirow{2}{*}{$\begin{array}{c}\text { Tipos de AF } \\
(\mathrm{T})\end{array}$} & $\mathrm{F}(5,281)$ & $F(1,285)$ & $F(1,285)$ & $\mathrm{F}(1,285)$ & $\mathrm{F}(1,285)$ & $\mathrm{F}(1,285)$ \\
\hline & 1,28 & 0,41 & 0,01 & $4,23 *$ & 0,44 & 0,05 \\
\hline \multirow{2}{*}{$\begin{array}{l}\text { Forma de } \\
\text { praticar }(\mathrm{F})\end{array}$} & $F(5,281)$ & $F(1,285)$ & $F(1,285)$ & $F(1,285)$ & $F(1,285)$ & $\mathrm{F}(1,285)$ \\
\hline & 1,54 & 2,51 & 0,10 & 0,15 & 2,13 & $5,80^{*}$ \\
\hline \multirow{2}{*}{$\begin{array}{l}\text { Tempo de } \\
\text { prática }(\mathrm{Te})\end{array}$} & $F(10,562)$ & $F(2,285)$ & $F(2,285)$ & $F(2,285)$ & $F(2,285)$ & $\mathrm{F}(2,285)$ \\
\hline & 1,49 & 2,54 & 0,39 & 1,37 & 2,19 & 0,36 \\
\hline $\mathrm{T} \times \mathrm{F} \times \mathrm{Te}$ & 0,60 & 0,94 & 0,23 & 1,65 & 1,58 & 1,77 \\
\hline
\end{tabular}

Notas: MANOVA $=$ análise de variância multivariada; ANOVA $=$ análise de variância univariada; $* * * p<0,001$;

Notas: DV: Diversão; SD: Saúde; AP: Aparência; CP: Competência e SC: Social.

Já no que se refere à forma de praticar, verificou-se que aqueles que praticavam $\mathrm{AF}$ acompanhados $(M=3,88 ; D P=0,15)$, faziam mais por motivos sociais do que aqueles que praticavam AF sozinhos $(M=3,32 ; D P=0,17)$. Resultado semelhante a este pode ser visto nos estudos de Moreno, Cervelló e Martínez (2007), onde verificaram que os sujeitos que praticavam AF em programas orientados tinham maiores motivos de diversão, saúde e competência, enquanto os que praticavam com os amigos tinham motivos mais relacionados ao fator social.

Em relação à variável tempo de prática, não foram encontrados resultados estatisticamente significativos. No entanto, sugere-se a realização de novos estudos para verificar a influência do tempo na motivação para praticar $\mathrm{AF}$, uma vez que se acredita que as pessoas que praticam atividade física há menos tempo sejam propícias a abandonar a prática regular de $\mathrm{AF}$, enquanto as pessoas que praticam há mais tempo possivelmente possuem menos chances de abandonar essa prática e assim manter hábitos saudáveis de vida. Por último, observou-se que o efeito de interação entre tipo de AF, forma de praticar e tempo de prática foi testado, no entanto, como visto na tabela acima, essas variáveis não interagiram.

No geral, verificou-se que os participantes deste estudo praticavam atividade física mais por motivos de saúde, diversão, aparência e competência e menos por motivos sociais, tendo em vista que apresentaram médias superiores ao ponto médio teórico da escala nesses quatro fatores, com exceção apenas do fator social. Entretanto, no geral, deve-se ressaltar que o fator saúde foi o que apresentou maior média. Destacase ainda que os participantes do sexo feminino e os idosos indicaram praticar atividade física mais por motivos de saúde do que os homens e os participantes mais jovens. Observa-se também que os participantes de exercício indicaram praticar AF mais por questões de aparência do que os praticantes de esporte e, ainda, aqueles que praticavam AF acompanhados o faziam mais por motivos sociais do que os que praticavam sozinhos. A seguir são apresentadas as conclusões da presente pesquisa.

\section{Conclusão}

Compreender o que motiva as pessoas a praticar AF tem sido um dos maiores desafios dos profissionais envolvidos com esta área, tendo em vista que algumas pesquisas indicam que apesar de se conhecerem os diversos benefícios associados à prática regular de $\mathrm{AF}$, o número de sedentários ainda é crescente e tem se tornado algo preocupante no que diz respeito à saúde da população brasileira (Andreoti \& Okuma, 2003; Epiphanio, 1999; Mello \& cols., 2005).

Nesse sentido, pode-se dizer que os resultados apresentados e discutidos anteriormente acerca dos motivos para se praticar AF e algumas variáveis sociodemográficas, permitem afirmar que os objetivos da presente pesquisa foram alcançados, pois a maior parte dos resultados foi coerente com os encontrados na literatura, bem como acredita-se que estudos desta natureza podem vir a contribuir com a construção do conhecimento nessa área, pois tais achados possibilitam uma maior compreensão acerca do que motiva as pessoas a praticar atividade física.

Entretanto, deve-se ter em mente que as variáveis aqui investigadas não são as únicas relevantes no estudo da motivação à prática de $\mathrm{AF}$, de modo que se sugerem novos estudos para verificar o papel de diferentes variáveis na 
motivação. A seguir, dispõem-se as principais limitações e sugestões de pesquisas futuras.

Algumas limitações da presente pesquisa são apontadas. Ressalta-se que, apesar da tentativa, nem todas as variáveis se mostraram balanceadas, apenas o sexo, que apresentou quase a mesma quantidade de homens $(50,8 \%)$ e mulheres $(49,2 \%)$; e a forma de prática, com quase o mesmo número de praticantes sozinhos $(47 \%)$ e acompanhados (53\%). Assim, novos estudos devem ser realizados buscando utilizar as variáveis de forma mais bem equilibrada.

Destaca-se, ainda, que não se contou com a colaboração de pessoas que praticavam AF em programas orientados, bem como não houve a participação de pessoas de todas as classes sociais, pois os participantes deste estudo foram abordados principalmente em bairros nobres da cidade do Natal, e a maioria em academias $(46,9 \%)$, local onde se paga para praticar AF. Dessa forma, sugere-se que em futuros estudos a variável renda também seja considerada, buscando contar com a participação de pessoas de todas as classes sociais e levando em consideração uma maior variabilidade de locais onde se pratica AF.

Acrescenta-se ainda que os resultados aqui obtidos não são generalizáveis para além da amostra considerada, pois, relembrando, tal como apontado no método, tratou-se de uma amostra não-probabilística. Assim, novos estudos poderiam ser úteis a fim de verificar a adequação dos resultados aqui observados. Apesar das limitações, acredita-se estar contribuindo para o acréscimo de dados empíricos à literatura brasileira sobre motivação à prática de atividades físicas. No entanto, futuras investigações considerando novas variáveis são necessárias na busca por melhor compreender a motivação à prática de AF e, assim, minimizar o risco ao sedentarismo.

\section{Referências}

Andreotti, M. C. \& Okuma, S. S. (2003). Perfil sócio-demográfico e de adesão inicial de idosos ingressantes em um programa de educação física. Revista Paulista de Educação Física, 17(2), 142-53.

Biddle, S. J. H. (1997). Current trends in sport and exercise psychology research. The Psychologist, 10(2), 63-69.

Caspersen, C. J., Powell, K. E. \& Christenson, G. M. (1985). Physical activity, exercise, and physical fitness: definitions and distinctions for health-relates research. Public Health Reports, 100(2) 172-179.

Célis-Merchán, A. G. (2006). Adaptación al Español de la escala revisada de Motivos para la Actividad Física (MPAM-R) y el Cuestionario de Clima Deportivo (SCQ). Avances en Medición, 4, 73-90.

Conselho Federal de Educação Física - CONFEF (2004). Atividade física: sinônimo de bemestar. Revista de Educação Física, 14. Obtido em 26 de junho de 2006 do World Wide Web: http://www.confef.org.br/.

Deci, E. L. \& Ryan, R. M. (1985). Intrinsic motivation and self-determination in human behavior. Nova Iorque: Plenum.

Dishman, R. K. (1994). Predicting and changing exercise and physical activity: what's practical and what's not. Em H. A. Quinney, L. Gauvin \& A. E. T. Wall (Orgs.). Toward active living: proceedings of the international conference on physical activity, fitness, and health (pp. 97-106). Toronto: Human Kinetics Publishers.

Epiphanio, E. H. (1999). Psicologia do esporte: apropriando e desapropriando. Psicologia Ciência e Profissão, 19(3), 70-73.

Fernandes, H. M. (2003). Motivação no contexto da educação física: estudo centrado no valor preditivo das intenções de prática desportiva em função da motivação intrínseca. Monografia, Universidade de Trásos-Montes e Alto Douro, Portugal.

Fernandes. H. M. \& Vasconcelos-Raposo, J. (2005). Continuum de auto-determinação: validade para a sua aplicação no contexto desportivo. Estudos de Psicologia, 10(3), 385-395.

Ferrareze, M. P. S. (1997). A influência da atividade física na melhora de qualidade de vida do homem. Dissertação de Mestrado, Universidade Estadual de Campinas, Campinas-SP.

Frederick, C. M. \& Ryan, R. M. (1993). Differences in motivation for sport and exercise and their relations with participation and mental health. Journal of Sport Behavior, 16(3), 125-145.

Gauvin, L. \& Spence, J. C. (1995). Psychological research on exercise and fitness: current research trends and future challenges. The Sport Psychologist, 9, 434-448.

Gonçalves, M. P. (2008). Adaptação e validação da escala de motivação à prática de atividades físicas. 
Dissertação de Mestrado, Universidade Federal do Rio Grande do Norte, Natal.

Gouveia, M. J. (2001). Tendências da investigação na psicologia do desporto, exercício e actividade física. Análise Psicológica, 19(1), 514.

Hair, J. F., Anderson, R. E., Tatham, R. L. \& Black, W. C. (2003). Multivariate data analysis. Nova Jersey: Prentice-Hall.

Hellín, P., Moreno, J. A. \& Rodriguez, P. L. (2004). Motivos de práctica físico-deportiva en la Región de Murcia. Cuadernos de Psicología del Deporte, 4(2), 101-116.

Howley, E. T. \& Don Franks, B. (2000). Manual do instrutor de condicionamento físico para a saúde $\left(3^{\mathrm{a}}\right.$ ed.). Porto Alegre: Artmed.

Kilpatrick, M. Hebert, E. \& Bartholomew, J. (2005). College estudantes' motivation for physical activity: differentiating men's and women's motives for sport participation exercise. Journal of American College Health, 54, 84-94.

Mello, M. T., Boscolo, R. A., Esteves, A. M. \& Tufik, S. (2005). O exercício físico e os aspectos psicobiológicos. Revista Brasileira de Medicina do Esporte, 11(3), 203-207.

Miragaya, A. (2006). Promoção da saúde através da atividade física. Em L. Da Costa (Org.). Atlas do esporte no Brasil: atlas do esporte, educação física e atividades físicas de saúde e lazer no Brasil (pp. 1628). Rio de Janeiro: CONFEF.

Moreno, J. A., Cervelló, E. \& González-Cutre, D. (2006). Motivación autodeterminada y flujo disposicional en el deporte. Anales de Psicologia, 22(2), 310-317.

Moreno, J. A., Cervelló, E. \& Martínez, A. (2007). Validación de la Escala de Medida de los Motivos para la Actividad Física - Revisada en españoles: diferencias por motivos de participación. Anales de Psicología, 23(1), $167-$ 176.

Ntoumanis, N. (2001). Empirical links between achievement goal theory and selfdetermination theory in sport. Journal of Sport Sciences, 19, 397-409.

Organização Mundial de Saúde - OMS (2006). Benefits of physical activity. Obtido em 20 de julho de 2006 do World Wide Web: http://www.who.int/dietphysicalactivity/facts heet_benefits/en/index.html.

Pérez, C. (2001). Técnicas estadísticas con SPSS. Madrid: Pratice Hall.

Ryan, R. M. \& Deci, E. L. (2000). Selfdetermination theory and the facilitation of intrinsic motivation, social development, and well-being. American Psychologist, 55(1), 68-78.

Ryan, R. M., Frederick, C. M., Lepes, D., Rubio, N. \& Sheldon, K. M. (1997). Intrinsic motivation and exercise adherence. International Journal of Sport Psychology, 28, 335-354.

Salles-Costa, R., Heilborn, M. L., Werneck, G. L., Faerstein, E. \& Lopes, C. S. (2003). Gênero e prática de atividade física de lazer. Cadernos de Saúde Pública, 19(2), 325-333.

Santos, S. C. \& Knijnik, J. D. (2006). Motivos de adesão à prática de atividade física na vida adulta intermediária. Revista Mackenzie de Educação Física e Esporte, 5(1), 23-34.

Schultz, D. P. \& Schultz, S. E. (2002). Teorias da Personalidade. São Paulo: Pioneira Thomson Learning.

Tabachnick, B. G. \& Fidell, L. S. (2001). Using multivariate statistics. Nova Iorque: Harper Collins College Publishers.

Recebido em agosto de 2008 Reformulado em setembro de 2009 Aprovado em dezembro de 2009 
Sobre os autores:

Marina Pereira Gonçalves é psicóloga pela Universidade Federal da Paraíba (UFPB) e mestre em Psicologia pela Universidade Federal do Rio Grande do Norte (UFRN). Atualmente é doutoranda em Psicologia Social na UFPB e professora assistente do curso de Psicologia da Universidade Federal do Vale do São Francisco (UNIVASF), onde ministra disciplinas de Psicometria e Avaliação Psicológica.

João Carlos Alchieri é professor adjunto da Universidade Federal do Rio Grande do Norte no PPG Psicologia e PPG Ciências da Saúde, com área de interesse em Avaliação Psicológica com ênfase em Instrumentos Psicológicos, Ensino de avaliação psicológica, Avaliação de personalidade, Saúde e Informatização de Medidas e Instrumentos. Atualmente é coordenador do GT Avaliação de Programas na ANPPEP e bolsista de produtividade. 